\title{
Synthesis and Electroanalytical Performance of a Composite Material Based on Poly $(3,4$-ethylenedioxythiophene) Doped with Lignosulfonate
}

\author{
Grzegorz Milczarek and Tomasz Rebis \\ Institute of Chemistry and Technical Electrochemistry, Poznan University of Technology, Piotrowo 3, 60-965 Poznan, Poland \\ Correspondence should be addressed to Grzegorz Milczarek, grzegorz.milczarek@put.poznan.pl
}

Received 15 June 2011; Accepted 4 October 2011

Academic Editor: Jiujun Zhang

Copyright ( $) 2012$ G. Milczarek and T. Rebis. This is an open access article distributed under the Creative Commons Attribution License, which permits unrestricted use, distribution, and reproduction in any medium, provided the original work is properly cited.

3,4-ethylenedioxythiophene (EDOT) was electropolymerized in the presence of sodium lignosulfonate (LS) at constant current density of $0.25 \mathrm{~mA} \mathrm{~cm}^{-2}$. As a result, a thin composite film consisting of poly(3,4-Ethylenedioxythiophene) and LS (PEDOT/LS) was deposited on the electrode surface. Unlike PEDOT, PEDOT/LS shows appreciable redox activity due to LS-derived quinone moieties with diffusion-like charge propagation across the film thickness. The film-modified gold electrodes can be used as voltammetric sensor of uric acid (UA) in the presence of ascorbic acid (AA). Interestingly, the UA response is catalysed by the presence of AA, and for high AA/UA concentration ratios more than 10-fold enhancement of the UA peak currents are apparent.

\section{Introduction}

Conducting polymers (CPs) have been attracting attention of material scientists and electrochemists for many years because of their easy preparation, high electroconductivity, as well as attractive electrochemical and optical properties [1]. Properties of CPs are to some extend a function of the counterion balancing the positive charge of the protonated conjugated polymer chains. Therefore, various sulfonated compounds have been successfully applied to dope conducting polymers and thus to tune their properties. Among those sulfonic acids [2], sulfonated phthalocyanines [3], sulfonated anthraquinone and benzoquinone derivatives $[4,5]$, and sulfonated polymers [6-9] are the most representative examples. On the other hand, lignosulfonates (LSs) obtainable in huge quantities as a byproduct of pulp and paper industry attracted rather little attention as dopants of CPs. For instance, Roy et al. [10] used lignosulfonate as polyanion to dope chemically synthesized polyaniline. Similarly LS was used as dopant for polypyrrole [11]. Other electrochemical applications of LSs were also reported. For example, Vagin et al. electropolymerized LS on steel and showed anticorrosion properties of thus formed thin film. The same authors also studied electrochemistry of lignosulfonate on glassy carbon and reported it not to undergo electropolymerization on this electrode [12]. Contrary to the latter observation, we electropolymerized LSs from an acidic electrolyte on preactivated glassy carbon and observed high redox activity of the formed coatings which could be practically applied in the electrocatalytic reduction of acidic nitrite [13] or electrocatalytic oxidation of NADH [14]. We also proved that LSs can be used as valuable electrolyte additives in electrochemical supercapacitors [15].

The aim of the present work is to investigate a possibility for electrochemical polymerization of 3,4 ethylenedioxythiophene EDOT in the presence of commercially available LS into thin redox-active films. With the ease of preparation and its low costs resulting from the usage of industrial byproducts, such films exhibiting valuable electrocatalytic properties could find widespread applications in the development of electrochemical sensing platforms.

\section{Experimental}

3,4-Ethylenedioxythiophene (EDOT), lignosulfonic acid (sodium salt) $\left(M_{w} 52,000\right)$, lithium perchlorate trihydrate, and 
acetonitrile (anhydrous, 99.8\%) were purchased from Aldrich. Uric acid (UA) (98\%) was provided by Fluka and ascorbic acid (AA) by POCH (Gliwice, Poland).

Before its modification, the working electrode (polycrystalline gold, $1.0 \mathrm{~mm}$ diameter) was polished with hydrophilic silica slurries of $0.05 \mu \mathrm{m}$ on Buehler polishing cloth with water as a lubricant, rinsed with water, and sonicated in a water bath for $5 \mathrm{~min}$.

Electrodeposition of PEDOT/LS composite films was performed by the modifying procedure commonly applied for unmodified PEDOT. A solution of $1 \mathrm{mg} \mathrm{cm}^{-3}$ EDOT and $1 \mathrm{mg} \mathrm{cm}^{-3}$ LS in $\mathrm{H}_{2} \mathrm{O} / \mathrm{ACN}(1 / 1, \mathrm{v} / \mathrm{v})$, containing $0.1 \mathrm{M}$ $\mathrm{LiClO}_{4}$ as supporting electrolyte, was used as the plating solution, and the polymerization was attained by applying a constant current density of $0.25 \mathrm{~mA} \mathrm{~cm}^{-2}$ for desired time. After modification, the PEDOT/LS-modified electrode was thoroughly rinsed with water and characterized electrochemically in phosphate buffer ( $\mathrm{pH} 7.4)$.

All the electrochemical measurements were carried out using a $\mu$ Autolab II electrochemical analyzer (EcoChemie, Utrecht-Netherlands) connected to a PC for control, data acquisition, and storage. A standard three-electrode configuration, consisting of the modified working, reference and counter electrodes, was incorporated into a glass cell (volume $20 \mathrm{~cm}^{3}$ ). The supporting electrolytes were degassed with a stream of purified nitrogen for $20 \mathrm{~min}$. prior to the measurements. The counter electrode was a platinum wire. All potentials reported in this paper are referenced to an $\mathrm{Ag} / \mathrm{AgCl}$ $(3 \mathrm{M} \mathrm{NaCl})$ electrode with no regard for the liquid junction potential.

XPS spectra of the composite film were obtained with a VSW photoelectron spectrometer (Vacuum Systems Workshop Ltd., England) using nonmonochromatized $\mathrm{Al} \mathrm{K} \alpha$ radiation $(1486.6 \mathrm{eV})$. The radiation source operated at $15 \mathrm{kV}$ and $14 \mathrm{~mA}$. The vacuum pressure was always kept around $3 \times 10^{-8}$ mbar. The binding energy scale was corrected by referring to the aromatic peak of the $\mathrm{C} 1 \mathrm{~s}$ spectrum as being $284.6 \mathrm{eV}$.

\section{Results and Discussion}

3.1. Electrochemical Polymerization of EDOT in the Presence of Lignosulfonate. As we have already proven, LSs undergo strong adsorption on electrodic surfaces, and, after initial oxidation, the adsorbates show characteristic redox activity assignable to reversible oxidation reduction of quinone moieties $[13,14]$. Unfortunately, the surface coverage of the redox sites was within submonolayer range. Therefore, one of the objectives of this part of the work was to optimize the composition of the deposited PEDOT/LS composite in order to force maximal activity of the LS-derived quinone groups.

Using the galvanostatic polymerization method described in experimental section, both $\mathrm{PEDOT} / \mathrm{ClO}_{4}$ and PEDOT/LS films were obtained as adherent and continuous deposits on the Au electrode. Figure 1 shows the voltage-time tracing during polymerization in the absence and presence of LS. As seen, the presence of LS macromolecules in the PEDOT-plating solution does not cause any appreciable

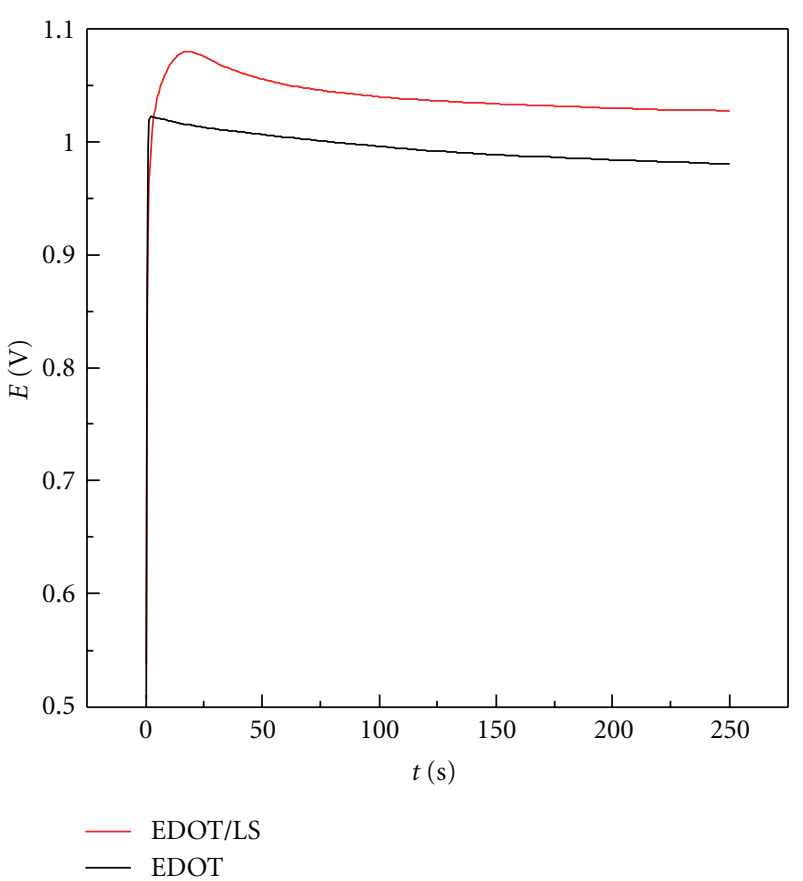

FIGURE 1: Voltage-time tracing recorded during galvanostatic polymerization of EDOT in the absence (black line) and presence of LS (red line).

change in the electrode polarization voltage during the deposition. The only visible change is that in the presence of LS at the concentration of $1 \mathrm{mg} \mathrm{cm}^{-3}$ the voltage curve is shifted up by ca. $50 \mathrm{mV}$. It is reasonable to assume that such a small change in electrode potential in not able to change substantially the characteristics of the PEDOT polymer formed in the presence of LS compared to its LS-free counterpart.

In contrast, the cyclic voltammograms of the obtained PEDOT/ClO 4 and PEDOT/LS films recorded in nearly neutral electrolyte show substantial differences (Figure 2).

The former shows typical capacitive behavior as reported for PEDOT and structurally similar polypyrrole, whereas the latter show a well-defined couple of peaks due to reaction mentioned above. This result clearly shows that the LS biomolecules are successfully incorporated into the growing PEDOT film and that their electrochemical activity is preserved. Unlike for LS alone electropolymerized on the surface coverage show much higher values being the function of LS concentration in PEDOT/LS-plating solution (Figure 3).

As seen the concentration of $1 \mathrm{mg} \mathrm{cm}^{-3}$ of LS provides the optimum conditions of LS incorporation into growing polymer layer judging from surface loading of quinone moieties and the fraction of redox charge contributing to the overall CV charge recorded. We also studied the effect of electrodeposition charge (time) on the two variables, and the results are presented in Figure 4. These plots show clearly that the loading of quinone moieties is nearly the linear function of the deposition charge, and, as opposite to LS polymerized on GC electrodes [14], the surface ladings can be tuned to reach values in the order of $10^{-8} \mathrm{~mol} \mathrm{~cm}^{-2}$. Besides, the efficiency of LS incorporation into the growing composite shows a peak for the polymerization charge of ca. $50 \mathrm{mC} \mathrm{cm}^{-2}$. 


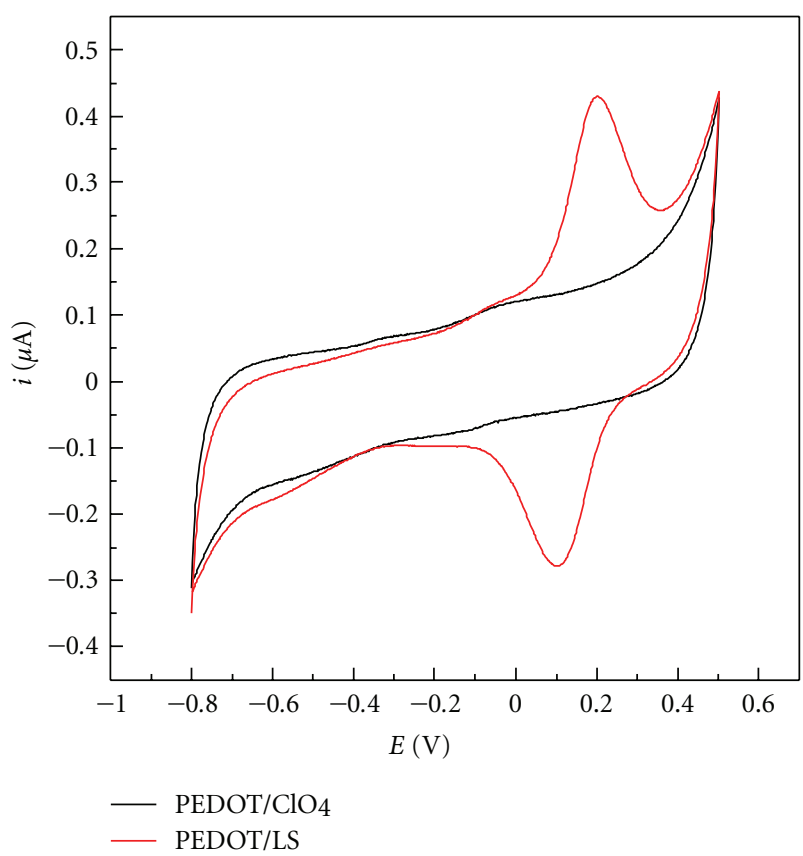

FIgUre 2: Comparison of cyclic voltammograms of PEDOT films electropolymerized in the absence (black line) and presence of LS (red line). LS concentration in the plating bath $-1 \mathrm{mg} \mathrm{cm}^{-3}$. Scan rate- $10 \mathrm{mV} \mathrm{s}^{-1}$. Electrolyte-phosphate buffer ( $\left.\mathrm{pH} 7.4\right)$.

Therefore, such a polymerization charge and LS concentration of $1 \mathrm{mg} \mathrm{cm}^{-3}$ were selected for further experiments.

3.2. XPS Analysis of the Composite Films. To give more insight into the composition of the PEDOT/LS polymer, the chemical structure of it was compared with $\mathrm{PEDOT} / \mathrm{ClO}_{4}$ by $\mathrm{X}$-ray photoelectron spectroscopy. Figure 5 shows the $\mathrm{O}(1 \mathrm{~s})$ and $S(2 p)$ core-level spectra for both types of polymers. The (1s) core level spectrum or PEDOT/CLO 4 and PEDOT/LS are depicted in Figures 5(a) and 5(c). Significant differences can be discerned between the $\mathrm{O}(1 \mathrm{~s})$ signals of the two kinds of samples. The rather unimodal $\mathrm{O}(1 \mathrm{~s})$ peak of $\mathrm{PEDOT} / \mathrm{ClO}_{4}$ contains the predominant feature at about $532.7 \mathrm{eV}$ attributed to the oxygen atom from the PEDOT chain together with the contribution from perchlorate anions and an almost negligible band at ca. $535.3 \mathrm{eV}$. The assignment of this band is currently unknown, but most probably it is due to adsorbed water molecules. On the other hand, the oxygen band of the LS-doped composite can be deconvoluted into two signals: the predominant one at BE of ca. 532.9 assigned to ethertype oxygen atoms in EDOT units and another one at BE of 531.5 due to oxygen atoms in sulfonic groups of LS. A similar doublet was observed for PEDOT doped with polystyrene sulfonate (PSS) [6] or toluene sulfonate [16].

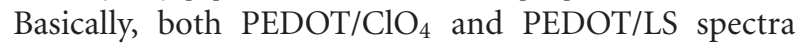
contain sulfur atoms of thiophene ring type manifested by a doublet of peaks at energy levels of 163.5 to $165 \mathrm{eV}$. For the former case, a minor contribution from a peak of higher binding energies is also noticed at $\mathrm{BE}$ of ca. $166.4 \mathrm{eV}$. Its origin may be assigned to the overoxidation of some number of thiophene rings resulting in oxygen attachment to sulfur

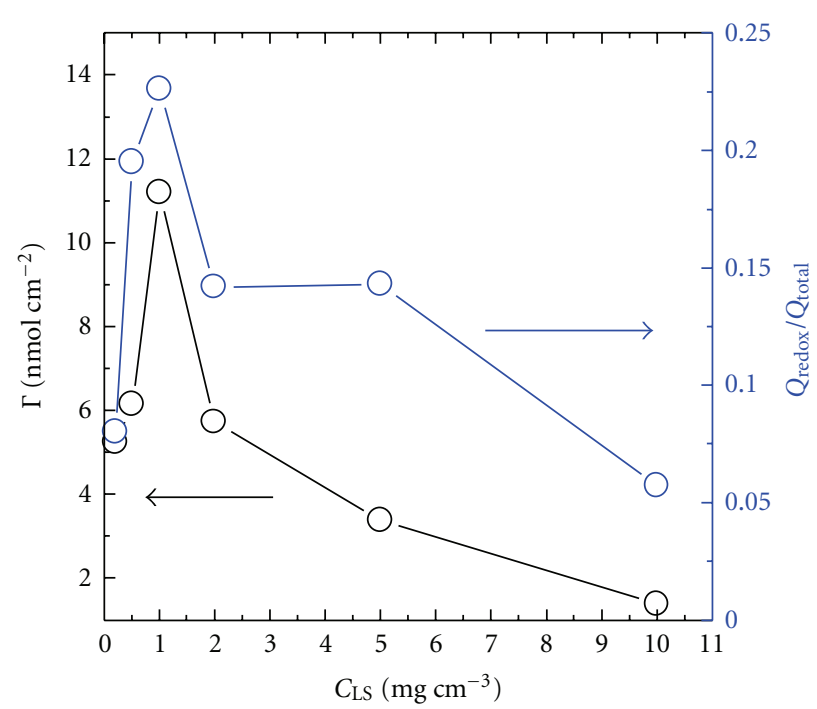

FIgURE 3: The effect of LS concentration on the redox activity of the PEDOT/LS composite expressed as surface coverage of quinone groups and the ratio of redox peaks charge versus total CV charge. The calculations were made from $\mathrm{CV}$ curves recorded at $10 \mathrm{mV} \mathrm{s}^{-1}$ in phosphate buffer ( $\mathrm{pH} 7.4)$.

atom [17]. On the other hand, for the PEDOT/LS composite, the sulfur spectra shows an additional well-defined peak at higher BE $(167.9 \mathrm{eV})$ due to incorporated LS molecules rich in sulfur atoms with three electronegative oxygens withdrawing the electrons from sulfur atom.

3.3. Electrochemical Characterization of the Composite Films. Figure 6 shows cyclic voltammograms recorded at various scan rates for the PEDOT/LS-modified gold electrode. As may be seen here, the voltammograms show a pair of redox peaks assignable to LS-derived quinone moieties. An analogous behavior was previously observed for LS electrodeposited on glassy carbon, but the calculated surface coverage was much lower than that observed for our composite [14]. This can be attributed to the presence of the PEDOT network acting as an electronically conducting grid transporting the electrons between the gold support and electroactive moieties of the biopolymer. The recorded peak currents are directly related to the potential scan rate, as expected for surface-anchored redox systems. In the selected range of scan rates, the peak potentials are virtually independent of scan rate, indicating facile electron transfer kinetics.

At higher scan rates, however, the dependence of peak currents versus sweep rate becomes proportional to the square root of scan rate indication diffusion-like transport of charge within the electroactive film. We used pulsed chronoamperometry to determine the apparent charge transport diffusion coefficient, and we found that for sufficiently short times the current decay response conforms to the Cottrell equation. From this dependence, for the film polymerized at $50 \mathrm{mC} \mathrm{cm}^{-2}$, we found the $\mathrm{D}_{\mathrm{ct}}$ value to be $1.6 \times 10^{-9} \mathrm{~cm}^{2} \mathrm{~s}^{-1}$.

3.4. Electroanalytical Application of the PEDOT/LS Composite Film. PEDOT-based materials have been reported to be 


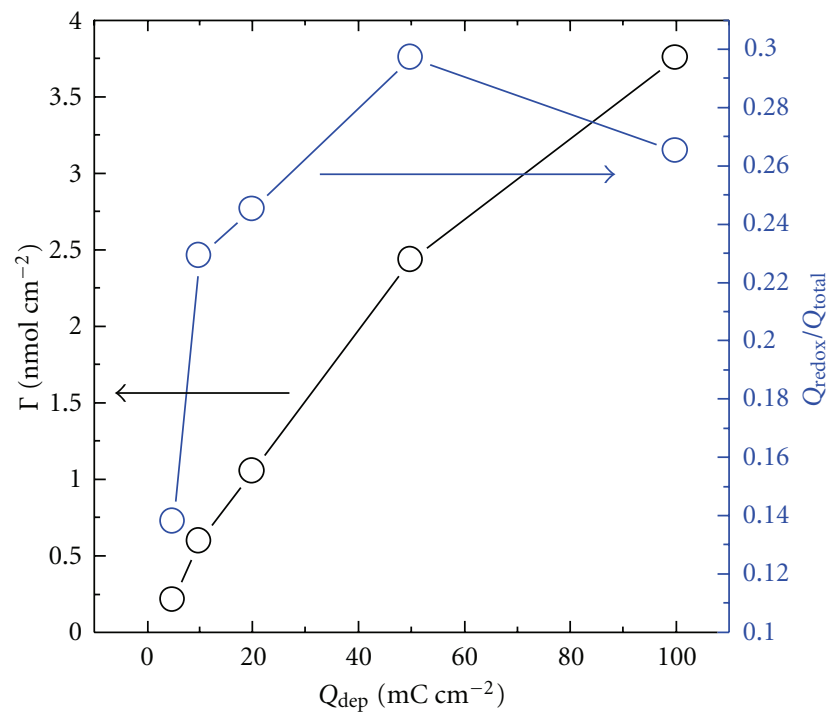

FIGURE 4: The effect of deposition charge on the redox activity of the PEDOT/LS composite expressed as surface coverage of quinone groups and the ratio of redox peaks charge versus total CV charge. The calculations were made from CV curves recorded at $10 \mathrm{mV} \mathrm{s}^{-1}$ in phosphate buffer ( $\mathrm{pH} 7.4)$.
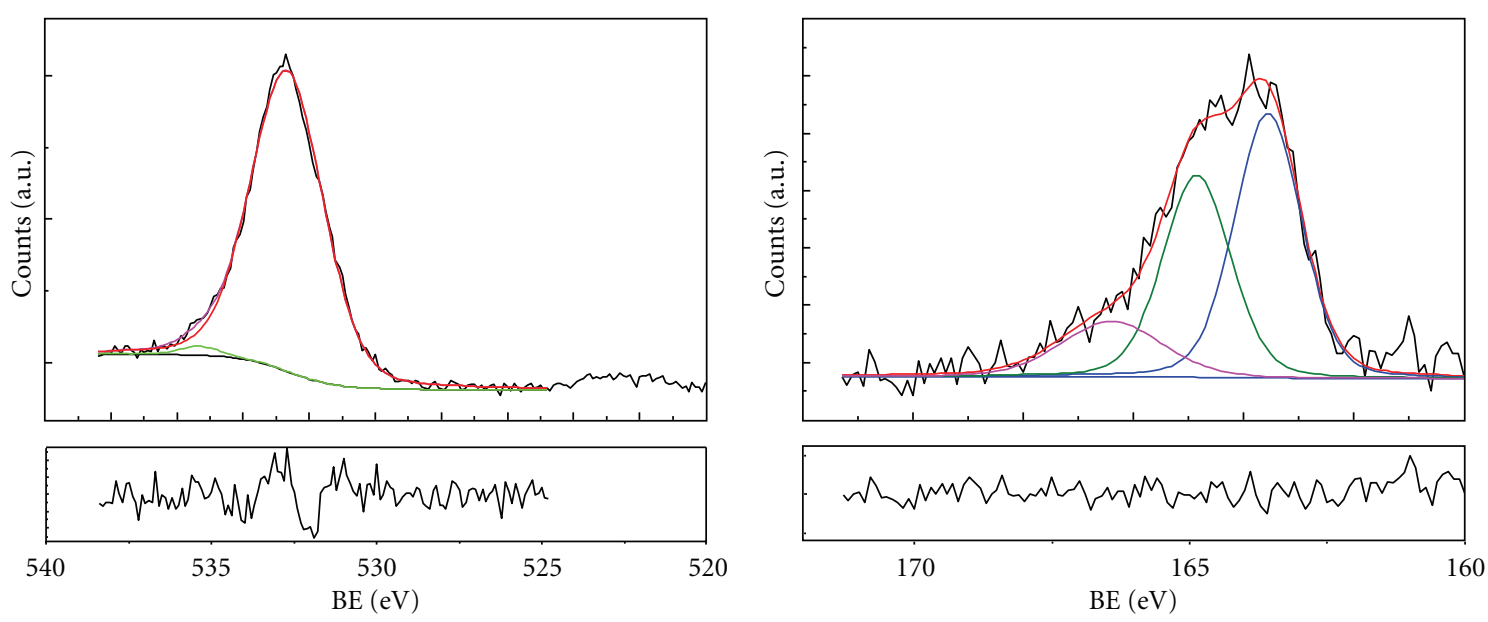

(a)

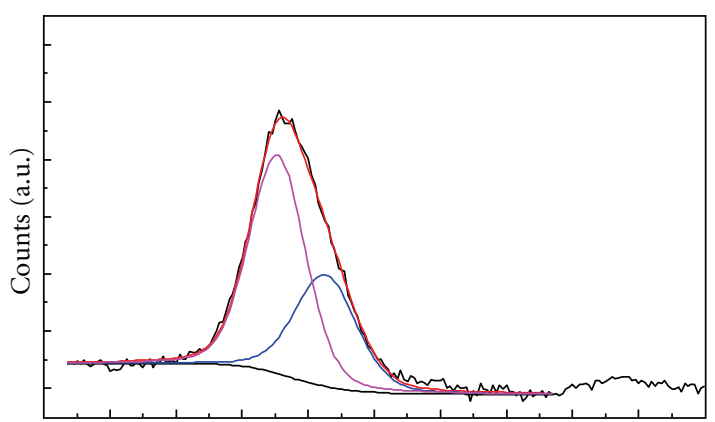

(b)
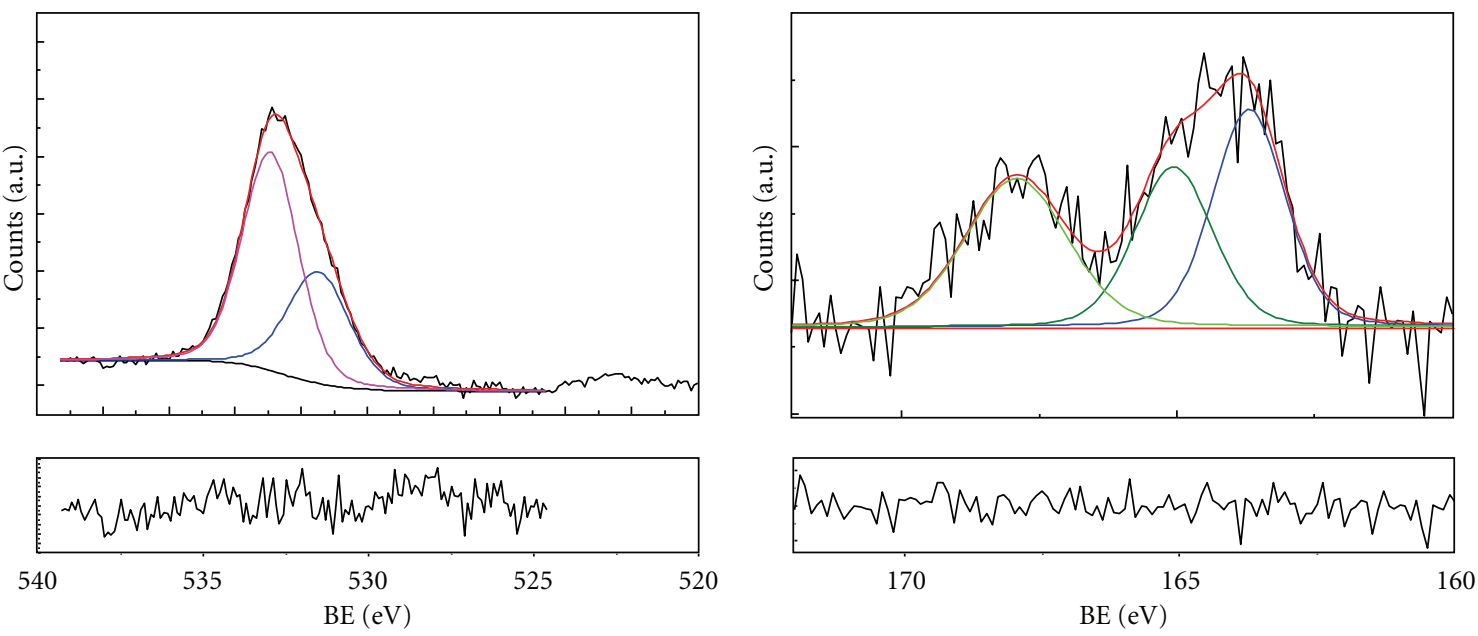

(c)

(d)

FIGURE 5: XPS core-level spectra of O(1s) (a, c) and S(2p) bands (c, d) for PEDOT/ClO 4 (a, b) and PEDOT/LS (c, d) composite. 


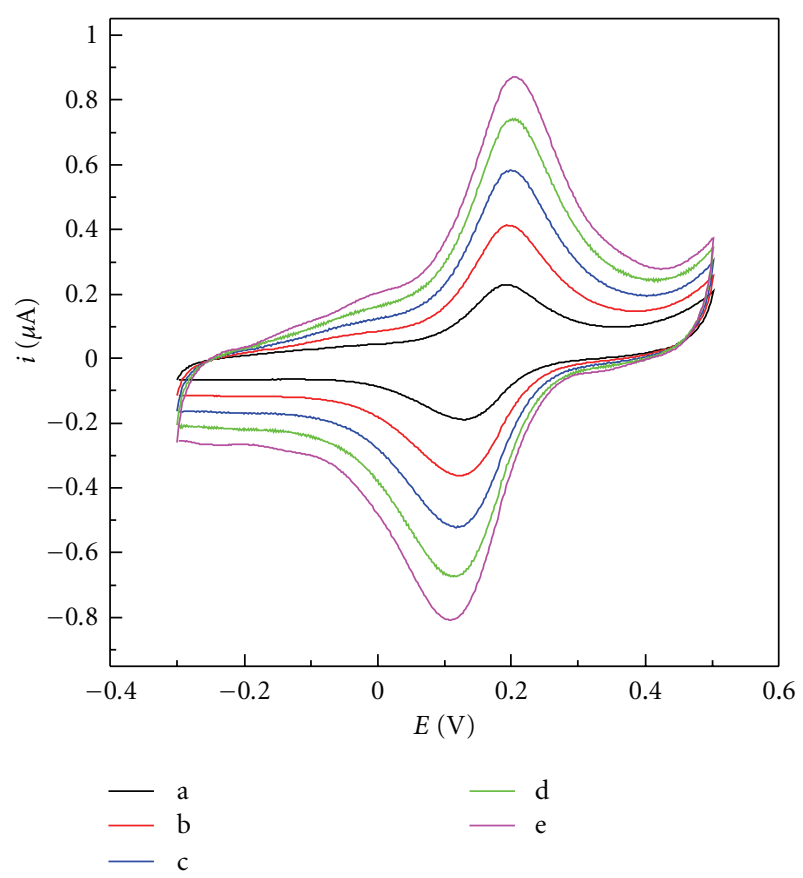

FIGURE 6: CV recorded on a PEDOT/LS-modified electrode in phosphate buffer ( $\mathrm{pH} 7.4$ ) at sweep rates of 10 (a), 20 (b), 30 (c), 40 (d), and $50 \mathrm{mV} \mathrm{s}^{-1}$ (e), respectively.

a good sensing platform for the electrochemical detection of various species, especially those of biological relevance. As a model system, we chose uric acid (UA) alone and in coexistence with ascorbic acid (AA). The two compounds are commonly found in body fluids, and the monitoring of UA concentration is of clinical importance. The problem is that usually the level of UA in the sample is much lower than that of AA. Besides, on common electrodic material, for example, gold or glassy carbon, both compounds discharge anodically at similar potentials. Therefore, improvements of the sensitivity of the working electrode toward UA have been becoming a long-standing issue of electroanalyses, and PEDOT films have been suggested as those showing valuable UA/AA response ratio $[18,19]$.

Figure 7 summarizes our preliminary data on the application of the PEDOT/LS-modified electrodes in the electrochemical detection of UA. Regarding the reports pointing at an electrocatalytic effect of ascorbic acid on the electrochemical detection of UA on PEDOT-modified electrodes [20], we measured the response to UA in the absence and presence of constant concentration of AA. At unmodified gold electrode, $\mathrm{UA}$ and AA give broad overlapped wave at potential region of $0.2-0.6 \mathrm{~V}$ (not shown). In contrast, as seen in Figure 7(a), UA gives a well-defined voltammetric peak at $0.33 \mathrm{~V}$ at submillimolar concentration range. The presence of $1 \mathrm{mM}$ AA in the background electrolyte itself does not generate any significant change in the voltammogram except that the surface redox peak of the PEDOT/LS composite is slightly enhanced. This is due to well-known catalytic activity of quinones in the mediated electrooxidation of AA [2]. The presence of AA does not affect peak position of the coexisting UA but increases it significantly (Figure 7(b)). The observed catalytic

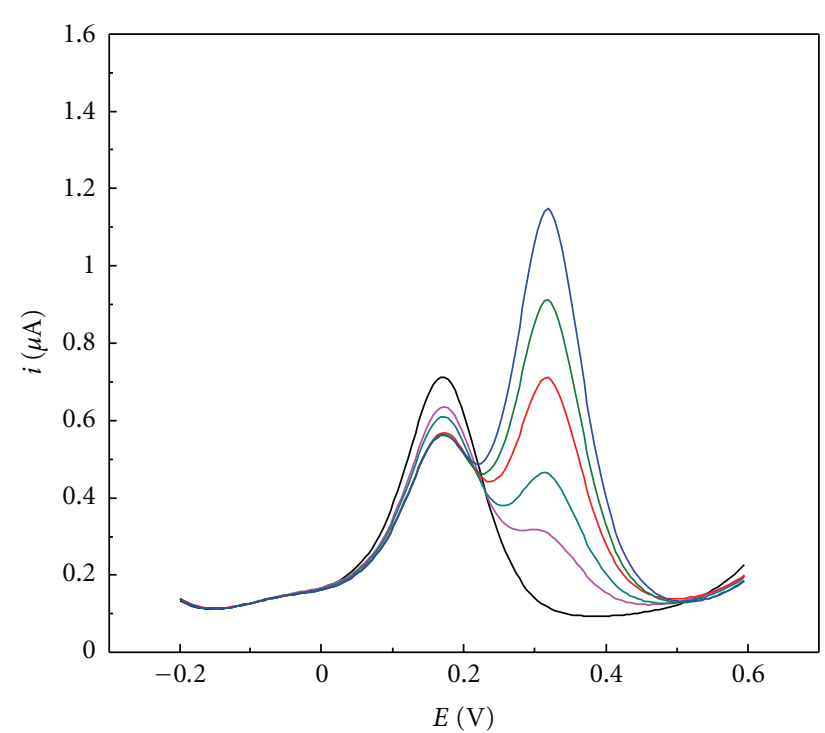

(a)

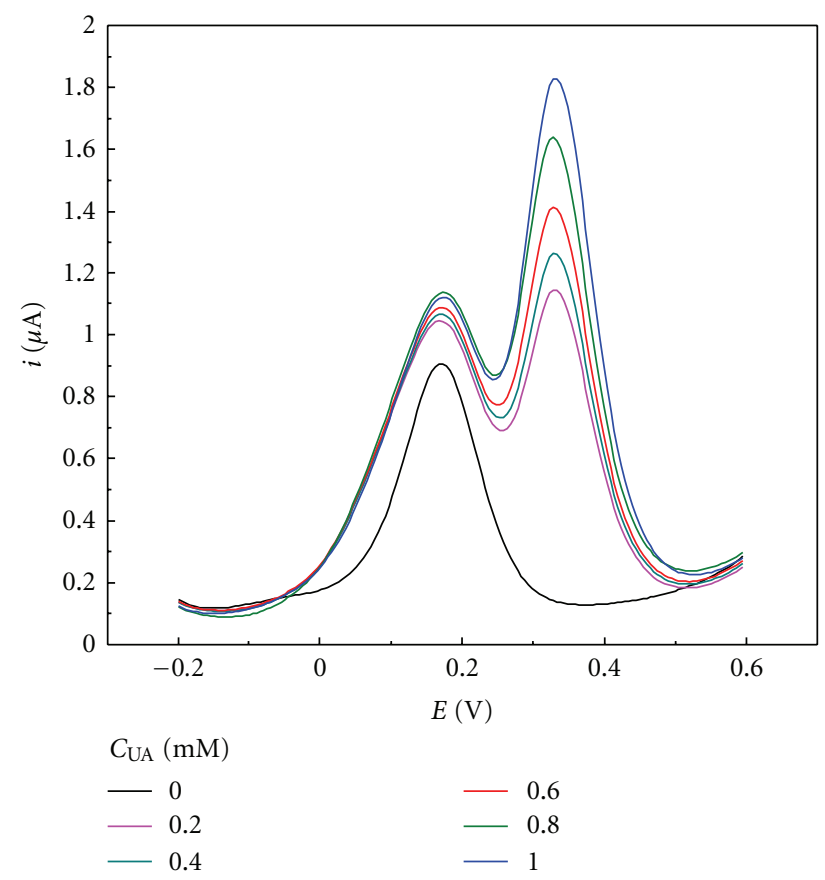

(b)

FIGURE 7: Differential pulse voltammograms of UA at the concentration increasing from 0 to $1.0 \mathrm{mM}(0.2 \mathrm{mM}$ step $)$ in the absence (a) and presence of $1 \mathrm{mM} \mathrm{AA} \mathrm{(b).}$

enhancement is a function of UA concentration or the ratio between AA and UA. This dependence is plotted in Figure 8. As seen, for low UA concentrations, the catalytic effect of AA may lead to over 10-fold enhancement of the UA peak current. Note that for the $\mathrm{PEDOT} / \mathrm{ClO}_{4}$ the effect is much less pronounced nor so much dependant on UA concentration. Even lower current enhancement was noticed for PEDOT polymerized in an organic medium; that is, $1 \mathrm{mM}$ AA caused the increased in the UA response from 77.5 to 86.2 $\mathrm{mA} \mathrm{mM}^{-1} \mathrm{~cm}^{-2}[20]$. 


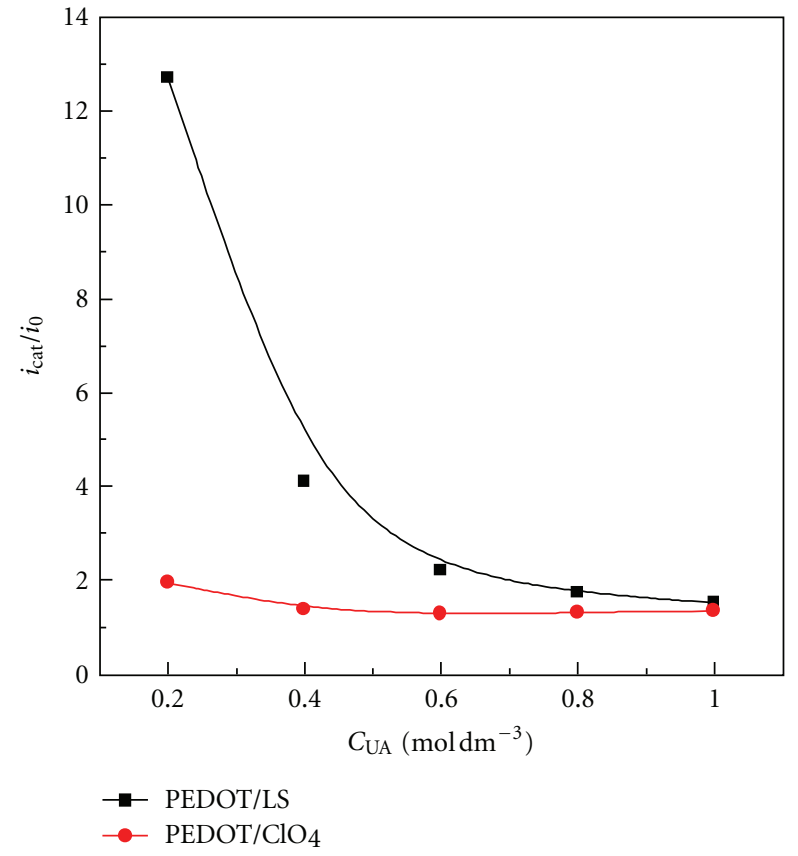

FIgURE 8: AA-induced peak current enhancement of UA on PEDOT/ClO 4 (red line) and PEDOT/LS film (black line) as a function of UA concentration.

\section{Concussion}

Electrodeposition of PEDOT in the presence of lignosulfonate has proved to give a redox active composite with quinone moieties. Electrode modification with such a composite film is a way to elaborate a voltammetric sensor for the assay of UA in the presence of coexisting AA. Interestingly, AA is found to enhance the response of UA on the PEDOT/LS film to a much stronger extent than on the LS-free PEDOT. Works are in progress to evaluate the performance of the proposed composite toward electrochemical sensing of other biomolecules.

\section{Acknowledgment}

This work was financially supported by the Polish Ministry of Science and Higher Education under project no. N N209 450939.

\section{References}

[1] T. A. Skotheim, R. L. Elsenbaumer, and J. R. Reynolds, Eds., Handbook of Conducting Polymers, Marcel Dekker, New York, NY, USA, 2nd edition, 1998.

[2] G. Milczarek, "Electrochemical modification of poly-aniline films in the presence of guaiacol-sulfonic acid," Electrochemistry Communications, vol. 9, no. 1, pp. 123-127, 2007.

[3] G. Milczarek, "Self-doped polyaniline films prepared by electropolymerization in the presence of sulfonated nickel phthalocyanine," Thin Solid Films, vol. 517, no. 21, pp. 6100-6104, 2009.
[4] X. Lang, Q. Wan, C. Feng et al., "The role of anthraquinone sulfonate dopants in promoting performance of polypyrrole composites as pseudo-capacitive electrode materials," Synthetic Metals, vol. 160, no. 15-16, pp. 1800-1804, 2010.

[5] N. Oyama, T. Sarukawa, Y. Mochizuki, T. Shimomura, and S. Yamaguchi, "Significant effects of poly(3,4-ethylenedioxythiophene) additive on redox responses of poly(2,5-dihydroxy1,4-benzoquinone-3,6-methylene) cathode for rechargeable Li batteries," Journal of Power Sources, vol. 189, no. 1, pp. 230239, 2009.

[6] J. Yan, C. Sun, F. Tan et al., "Electropolymerized poly(3,4ethylenedioxythiophene):poly(styrene sulfonate) (PEDOT: PSS) film on ITO glass and its application in photovoltaic device," Solar Energy Materials and Solar Cells, vol. 94, no. 2, pp. 390-394, 2010.

[7] C. Y. Li, T. C. Wen, and T. F. Guo, "Sulfonated poly(diphenylamine) as a novel hole-collecting layer in polymer photovoltaic cells," Journal of Materials Chemistry, vol. 18, no. 37, pp. 4478-4482, 2008.

[8] Z. Mousavi, T. Alaviuhkola, J. Bobacka, R. M. Latonen, J. Pursiainen, and A. Ivaska, "Electrochemical characterization of poly(3,4-ethylenedioxythiophene) (PEDOT) doped with sulfonated thiophenes," Electrochimica Acta, vol. 53, no. 11, pp. 3755-3762, 2008.

[9] T. Kim, J. Kim, Y. Kim, T. Lee, W. Kim, and K. S. Suh, "Preparation and characterization of poly(3,4-ethylenedioxythiophene) (PEDOT) using partially sulfonated poly(styrenebutadiene-styrene) triblock copolymer as a polyelectrolyte," Current Applied Physics, vol. 9, no. 1, pp. 120-125, 2009.

[10] S. Roy, J. M. Fortier, R. Nagarajan et al., "Biomimetic synthesis of a water soluble conducting molecular complex of polyaniline and lignosulfonate," Biomacromolecules, vol. 3, no. 5, pp. 937-941, 2002.

[11] C. Yang and P. Liu, "Water-dispersed conductive polypyrroles doped with lignosulfonate and the weak temperature dependence of electrical conductivity," Industrial and Engineering Chemistry Research, vol. 48, no. 21, pp. 9498-9503, 2009.

[12] M. Y. Vagin, S. A. Trashin, and A. A. Karyakin, "Corrosion protection of steel by electropolymerized lignins," Electrochemistry Communications, vol. 8, no. 1, pp. 60-64, 2006.

[13] G. Milczarek, "Lignosulfonate-modified electrode for electrocatalytic reduction of acidic nitrite," Electroanalysis, vol. 20, no. 2, pp. 211-214, 2008.

[14] G. Milczarek, "Lignosulfonate-modified electrodes: electrochemical properties and electrocatalysis of NADH oxidation," Langmuir, vol. 25, no. 17, pp. 10345-10353, 2009.

[15] G. Lota and G. Milczarek, "The effect of lignosulfonates as electrolyte additives on the electrochemical performance of supercapacitors," Electrochemistry Communications, vol. 13, no. 5, pp. 470-473, 2011.

[16] F. Petraki, S. Kennou, S. Nespurek, and M. Biler, "A spectroscopic study for the application of a PEDOT-type material as buffer layer in electronic devices," Organic Electronics: Physics, Materials, Applications, vol. 11, no. 8, pp. 1423-1431, 2010.

[17] P. Tehrani, A. Kanciurzewska, X. Crispin, N. D. Robinson, M. Fahlman, and M. Berggren, "The effect of $\mathrm{pH}$ on the electrochemical over-oxidation in PEDOT:PSS films," Solid State Ionics, vol. 177, no. 39-40, pp. 3521-3527, 2007.

[18] S. S. Kumar, J. Mathiyarasu, K. L. Phani, Y. K. Jain, and V. Yegnaraman, "Determination of uric acid in the presence of ascorbic acid using poly(3,4-ethylenedioxythiophene)-modified electrodes," Electroanalysis, vol. 17, no. 24, pp. 2281-2286, 2005. 
[19] F. Sekli-Belaidi, P. Temple-Boyer, and P. Gros, "Voltammetric microsensor using PEDOT-modified gold electrode for the simultaneous assay of ascorbic and uric acids," Journal of Electroanalytical Chemistry, vol. 647, no. 2, pp. 159-168, 2010.

[20] F. Sekli-Belaidi, D. Evrard, and P. Gros, "Evidence of an EC' mechanism occurring during the simultaneous assay of ascorbic and uric acids on poly(3,4-ethylenedioxythiophene) modified gold microsensor," Electrochemistry Communications, vol. 13, no. 5, pp. 423-425, 2011. 


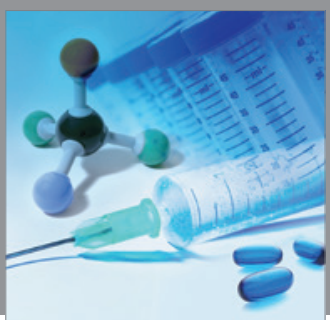

International Journal of

Medicinal Chemistry

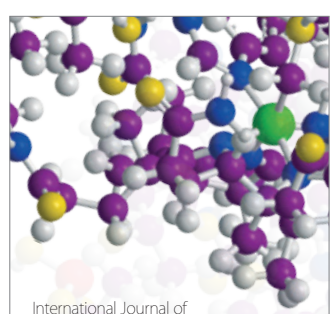

Carbohydrate Chemistry

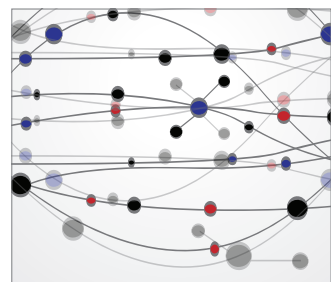

The Scientific World Journal
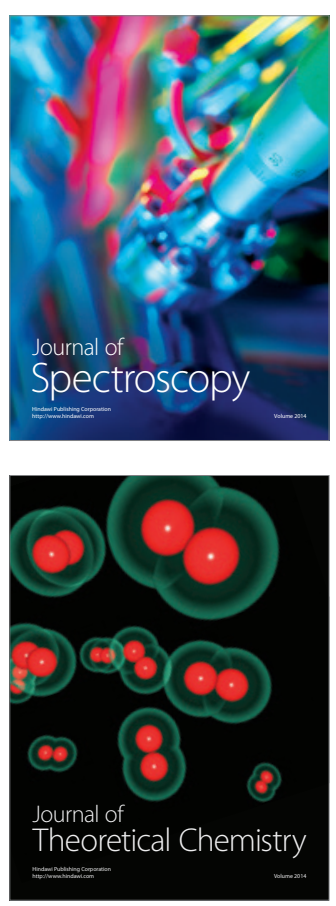
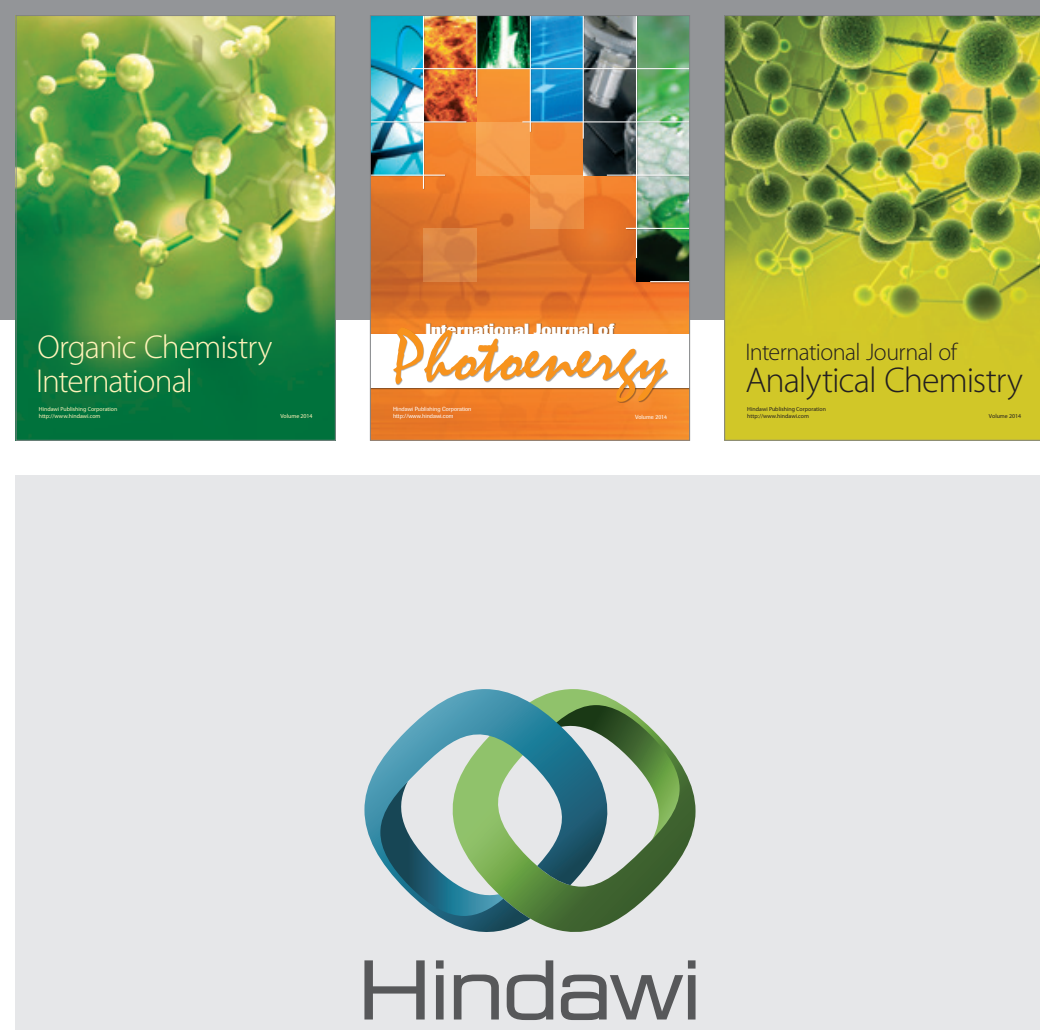

Submit your manuscripts at

http://www.hindawi.com
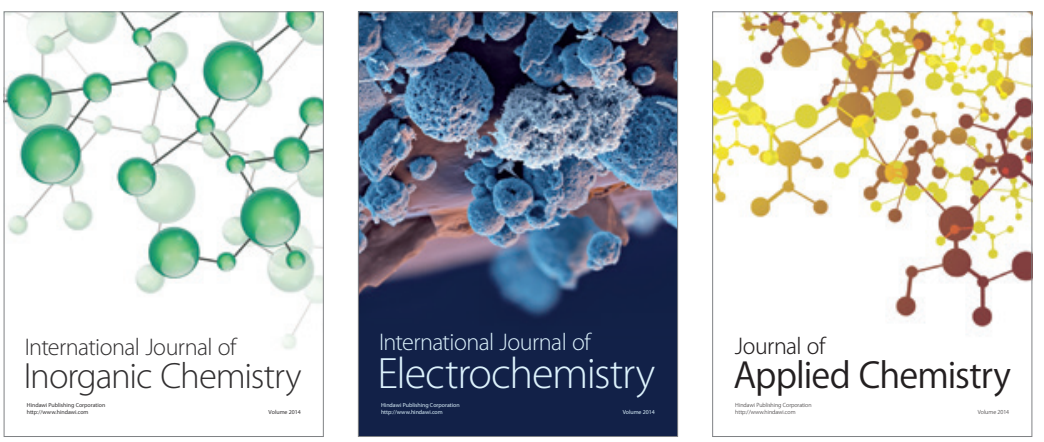

Journal of

Applied Chemistry
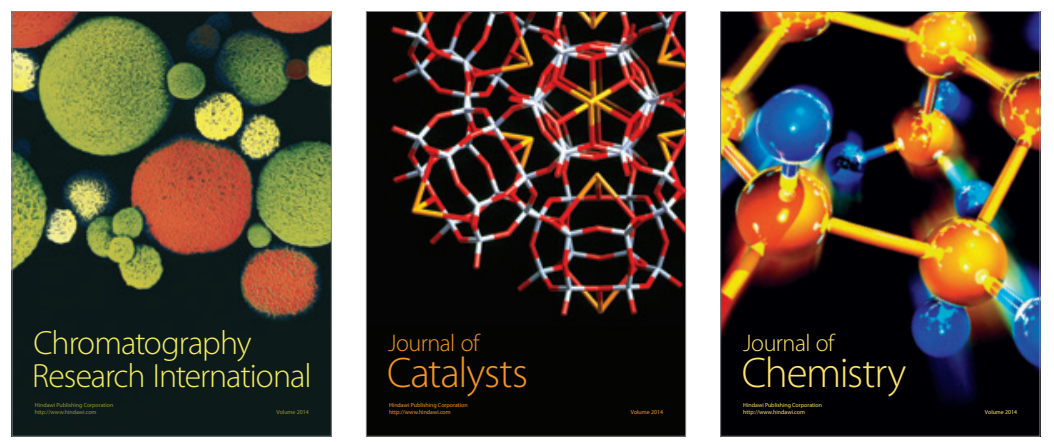
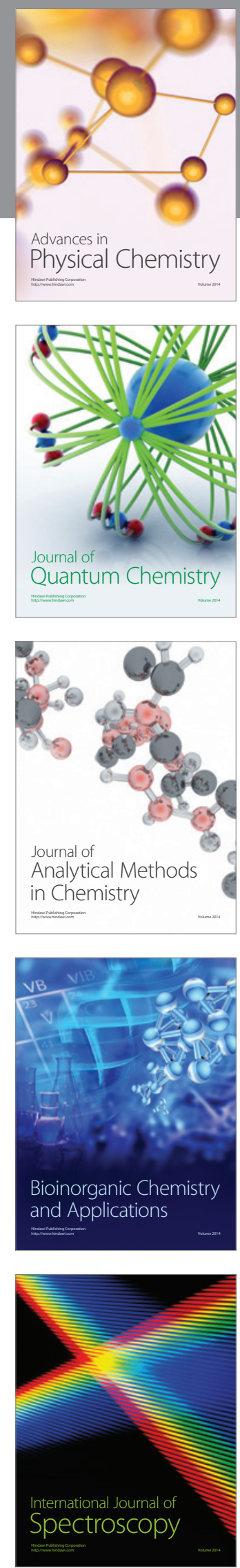